\title{
Irreversible Investment in Alternative Projects
}

\author{
Jean-Paul Décamps* Thomas Mariotti ${ }^{\dagger} \quad$ Stéphane Villeneuve ${ }^{\ddagger}$
}

First draft: 16 June 2003

\begin{abstract}
We examine the problem of a risk-neutral investor who has to choose among two alternative projects of different scales under output price uncertainty. We show that as soon as investment in the smaller scale project is sometimes optimal, the optimal investment strategy is not a trigger strategy and the optimal investment region is dichotomous. Whenever the investor has the opportunity to switch from the smaller scale to the larger scale project, the dichotomy of the investment region can persist even when uncertainty becomes large.

Keywords: Investment under Uncertainty, Optimal Stopping. JEL Classification: C61; D83.
\end{abstract}

${ }^{*}$ Université de Toulouse (GREMAQ-IDEI), Manufacture des Tabacs, 21, Allée de Brienne, 31000 Toulouse, France. Email: decamps@cict.fr.

${ }^{\dagger}$ Department of Economics, London School of Economics and Political Science, Houghton Street, WC2A 2AE, United Kingdom, and Université de Toulouse (CNRS-GREMAQ-IDEI), Manufacture des Tabacs, 21, Allée de Brienne, 31000 Toulouse, France. Email: t.mariotti@lse.ac.uk.

¥Université de Toulouse (GREMAQ), Manufacture des Tabacs, 21, Allée de Brienne, 31000 Toulouse, France. Email: stephane-villeneuve@univ-tlse1.fr. 


\section{INTRODUCTION}

The literature on irreversible investment pioneered by Arrow and Fisher (1974) and Henry (1974) is based on the premise that there are three factors driving the investment decision. First, there is some uncertainty about the future cash-flows of the investment project. Next, investment is at least partially irreversible, in the sense that investment expenditures cannot be fully recovered. Last, there is some flexibility in the timing of investment, which is valuable because it gives the investor the option to wait for new information. As a result, the loss of this option value at the time the firm invests represents an additional opportunity cost of investment, and investment options are exercised significantly above the point at which expected discounted cash-flows cover the sunk investment expenditures.

In the benchmark case of a single indivisible project, the optimal investment policy can be mathematically determined as the solution of an optimal stopping problem. The prototype of this approach is the model of McDonald and Siegel (1986), in which the underlying value of the investment project evolves as a geometric Brownian motion. Under this formulation, the optimal investment strategy is a trigger strategy. Specifically, the investment option should be exercised at the first time where the value of the investment project exceeds a critical threshold that can be explicitly computed using standard smooth-fit techniques (Dixit and Pindyck (1994)).

The large and rapidly growing literature on investment under uncertainty has also recognized as key feature of investment decisions the sequential nature of investment (Majd and Pindyck (1987), Bar-Ilan and Strange (1998)), the importance of entry and exit decisions (Dixit (1989)), and addressed issues like incremental capacity choice (Pindyck (1988), Kandel and Pearson (2002)), costly reversibility (Abel and Eberly (1996)), and technology adoption (Farzin, Huisman and Kort (1998)).

In this short paper, we leave aside these complex and meaningful extensions of the theory and revisit an old standard question, namely the choice of mutually exclusive investment projects under uncertainty. The starting point of our analysis is the model of Dixit (1993). In this paper, Dixit examines the problem of an investor who has to choose one project among multiple projects of different scales. The instantaneous profit generated by each investment project is a linear function of a single geometric Brownian motion, and projects with larger sunk investment costs are associated with larger profits. At the time of the investment, the investor selects the project with the larger net present value, and thus the underlying payoff function of the investor is the upper envelope of the family of affine functions representing the net present values of investing in each project.

Dixit's (1993) treatment of this problem relies on a simple adaptation of the oneproject case studied by McDonald and Siegel (1986). Namely, he argues that each project can be evaluated separately, and the solution to the investor's problem is simply 
to choose the project with the highest option value. As an illustration, suppose there are two projects, $G$ and $N$. One could for instance think of these two projects as alternative ways of producing electricity, by using a gas or a nuclear technology. Project $G$ has lower investment cost and generates lower profits than project $N$. Separate evaluation of these two projects leads to two option values, and associated critical thresholds $p_{G}$ and $p_{N}$ for the underlying geometric Brownian motion of output prices that drives the instantaneous profit of each project and is the relevant state variable. According to Dixit (1993), the solution of the investor's problem is to invest in the project that generates the highest option value, when the corresponding threshold $p_{G}$ or $p_{N}$ is reached. For values of the output price greater than this optimal threshold, investment is immediate and the project with the highest net present value is selected.

This is precisely this last point that we believe is not correct in Dixit's (1993) argument. Suppose that the lower cost project $G$ generates in itself a higher option value than the larger cost project $N$. In particular, when the current value of the output price is $p_{G}$, the investor is strictly better off investing in $G$ than in $N$. This implies that $p_{G}$ is lower than the indifference point at which the net present values of the two projects are equal. The key point is that it is never optimal to invest in either project when the current value of the output price is at this indifference point. The reason is that the payoff function of the investor, i.e., the upper envelope of the net present values of investing in each project, exhibits an upward derivative jump at this point. Using a local time argument, we show that this implies the optimality of delaying investment around this indifference point.

As a result, when the option of investing in $G$ is higher than that of investing in $N$, the optimal investment policy is no longer a trigger strategy and the optimal investment region in the state space is not connected. Instead, there is a dichotomy of the investment region. Specifically, there are two critical thresholds $p_{L}$ and $p_{R}$ around the indifference point such that if the current value of the output price lies between $p_{G}$ and $p_{L}$, it is optimal to invest in $G$, while if the current value of the output price is above $p_{R}$, it is optimal to invest in $N$. The intermediate region between $p_{L}$ and $p_{R}$ is an inaction region in which the investor waits to see in which project to invest. If the output price raises above $p_{R}$, it is optimal to invest in $N$, while if it drops down to $p_{L}$, it is optimal to invest in $G$.

The existence of this intermediate inaction region implies that, in striking contrast with most standard real options models, it can be optimal to invest in a project even though the instantaneous profit flow associated to this project falls. Investing in $G$ when the output price crosses down the threshold $p_{L}$ is optimal because $p_{L}$ is higher than the threshold $p_{G}$ above which it would be optimal to invest in $G$ if that were the only investment option available, and because it would be too costly to wait until the threshold $p_{R}$ is reached to invest in $N$-in other terms, "a bird in the hand is worth 
two in the bush." Moreover, there is a region of the state space in which it is optimal to delay investment while it would be optimal to invest if only one project, $N$ or $G$, were available. This illustrates the interaction between the two investment options, which is not taken into account in Dixit's (1993) solution. Adding a new investment option to an existing one increases the demand for information and creates an additional incentive to delay investment, even if, when evaluated separately, the second option is dominated by the first.

To illustrate these points, we consider a slightly modified version of Dixit's (1993) model in which the investor has the option to switch from project $G$ to project $N$ by incurring the corresponding sunk cost. (Switching from $N$ to $G$ is clearly suboptimal as the instantaneous profit under $G$ is lower than under $N$.) This implies that the expected payoff from investing in $G$ is itself an option value. This extension is motivated by the natural question of whether the dichotomy of the optimal investment region is a robust phenomenon, and, more specifically, of whether greater uncertainty systematically leads to the adoption of larger projects. In Dixit's (1993) original model, the option of investing in the larger cost project $N$ always dominates that of investing in the lower cost project $G$ if the volatility of the output price process is high enough. It is then never optimal to invest in $G$, and the solution to the investor's problem is to invest in $N$ as soon as the output price exceeds the critical threshold $p_{N}$. Thus when the option to switch from the smaller cost project to the larger cost project is not present, the dichotomy of the investment region disappears as uncertainty becomes large. By contrast, we show that, whenever the investor has the option to switch from $G$ to $N$, there are parameter values such that the dichotomy of the optimal investment region will be preserved and investment in $G$ will occur with positive probability even for a very high volatility of the underlying process.

The paper is organized as follows. Section 2 describes the model and delineates parameter restrictions ensuring the existence of a dichotomous investment region. Our main result is derived in Section 3.

\section{An Investment Problem \\ 2.1. The Model}

Our model is adapted from Dixit's (1993) model of choice among alternative discrete projects under uncertainty. We simplify his model by assuming that only two alternative project choices are available. Unlike him, however, we do not assume that the project choice is irreversible.

Time is continuous, and labelled by $t \geq 0$. There is a single risk-neutral investor who can engage in one of two projects, $G$ or $N$, of different scales. The investor can operate only one project at a time. Project $N$ is a larger scale project that generates 
a higher output than project $G$. Specifically, investment in project $k$ entails a sunk capital cost $I_{k}$, where $I_{N}>I_{G}$, and generates an output flow $x_{k}$, where $x_{N}>x_{G}$. A project once installed incurs no operating costs. The instantaneous profit generated by project $k$ is $x_{k} P_{t}$, where $P=\left\{P_{t} ; t \geq 0\right\}$ is a geometric Brownian motion with drift $\mu$ and volatility $\sigma$ :

$$
\mathrm{d} P_{t}=\mu P_{t} \mathrm{~d} t+\sigma P_{t} \mathrm{~d} W_{t} ; \quad t \geq 0,
$$

that represents the dynamics of output price. The investor discounts future revenue and costs at a constant rate $r>\mu$. A project once installed can last forever, but the investor can switch from one project to the other by incurring the corresponding sunk cost. Since the larger scale project $N$ generates higher profits than the lower scale project $G$ once it is in place, it is clear that it is never optimal to switch from $N$ to $G$. Hence, when the current value of the output price $P$ is $p$, the gross expected discounted profits of investing in project $N$ are given by:

$$
v_{N}(p)=\frac{x_{N}}{r-\mu} p
$$

On the other hand, whenever project $G$ has been adopted, it is optimal to switch to project $N$ whenever the output price $P$ becomes large enough. Specifically, when the current value of $P$ is $p$, the gross expected discounted profits of investing in project $G$ are given by:

$$
v_{G}(p)=\sup _{\tau \in \mathcal{T}^{P}} \mathbb{E}\left[\int_{0}^{\tau} \mathrm{e}^{-r t} P_{t}^{p} x_{G} \mathrm{~d} t+\mathrm{e}^{-r \tau}\left(v_{N}\left(P_{\tau}^{p}\right)-I_{N}\right)\right],
$$

where $\mathcal{T}^{P}$ is the set of stopping times adapted to the filtration generated by $P$, and the superscript $p$ in $P_{t}^{p}$ reflects the dependence of $P$ on its initial value $p .{ }^{1}$ It is well-known that the solution to this problem consists to switch from $G$ to $N$ as soon as the output price $P$ exceeds the critical threshold:

$$
p_{G N}=\frac{\beta}{\beta-1} \frac{I_{N}(r-\mu)}{x_{N}-x_{G}},
$$

where $\beta=1 / 2-\mu / \sigma^{2}+\sqrt{\left(1 / 2-\mu / \sigma^{2}\right)^{2}+2 r / \sigma^{2}}>1$ is the positive root of the quadratic equation $\xi(\xi-1) \sigma^{2} / 2+\mu \xi-r=0$. Standard computations then leads to the following explicit expression for $v_{G}$ :

$$
v_{G}(p)= \begin{cases}\frac{x_{G}}{r-\mu} p+\left(\frac{p}{p_{G N}}\right)^{\beta} \frac{I_{N}}{\beta-1} & \text { if } p \leq p_{G N}, \\ \frac{x_{N}}{r-\mu} p-I_{N} & \text { if } p>p_{G N} .\end{cases}
$$

\footnotetext{
${ }^{1}$ In Dixit's (1993) model, the option to switch from $G$ to $N$ is not available, and both $v_{G}$ and $v_{N}$ are linear functions, with slopes $x_{G} /(r-\mu)$ and $x_{N} /(r-\mu)$ respectively.
} 
(See, e.g., Dixit and Pindyck (1994).) A simple but useful remark is that $v_{G} \leq v_{N}$, with equality at zero only. The reason is that the two functions coincide at zero, and that $v_{G}$ is convex with a slope that is smaller or equal than the slope of $v_{N}$ everywhere. (Note that the two functions are linear with the same slope on $\left[p_{G N}, \infty\right)$.) The intuition for this is clear: the gross expected discounted profits generated by the larger scale project $N$ are greater than those generated by $G$, taking into account the opportunity to invest eventually at cost $I_{N}$ in project $N$.

The investor's problem can now be written as:

$$
V(p)=\sup _{\tau \in \mathcal{T}^{P}} \mathbb{E}\left[\mathrm{e}^{-r \tau} v\left(P_{\tau}^{p}\right)\right]
$$

where the payoff function $v$ is the upper envelope of $v_{G}-I_{G}$ and $v_{N}-I_{N}$ :

$$
v(p)=\max \left\{v_{G}(p)-I_{G}, v_{N}(p)-I_{N}\right\} ; \quad p \geq 0 .
$$

It should be noted that $v_{G}(0)-I_{G}>v_{N}(0)-I_{N}$ and $v_{G}(p)-I_{G}<v_{N}(p)-I_{N}$ for $p>p_{G N}$. Again, the slope of $v_{G}-I_{G}$ is always less than the constant slope of $v_{N}-I_{N}$. Hence there exists a unique price $\tilde{p}<p_{G N}$ such that $v_{G}(p)-I_{G} \geq v_{N}(p)-I_{N}$ if and only if $p \leq \tilde{p}$, with equality if $p=\tilde{p}$. In what follows, we shall refer to $\tilde{p}$ as the indifference point. A key feature of the payoff function $v$ is that it is not differentiable at the indifference point $\tilde{p}$, with $v_{p}\left(\tilde{p}^{+}\right)=v_{N p}(\tilde{p})>v_{G p}(\tilde{p})=v_{p}\left(\tilde{p}^{-}\right)$

\subsection{Two Auxiliary Problems}

As benchmarks, it will be helpful to determine the solution to two auxiliary investment problems. Suppose first that one can only invest in project $N$. The investor's problem is then:

$$
V_{N}(p)=\sup _{\tau \in \mathcal{T}^{P}} \mathbb{E}\left[\mathrm{e}^{-r \tau}\left(v_{N}\left(P_{\tau}^{p}\right)-I_{N}\right)\right]
$$

whose solution consists to invest in $N$ as soon as the output price $P$ exceeds the critical threshold:

$$
p_{N}=\frac{\beta}{\beta-1} \frac{I_{N}(r-\mu)}{x_{N}} .
$$

Suppose next that investment is constrained to be sequential, with the investment in $G$ occurring before that in $N$. The investor's problem is then:

$$
V_{G}(p)=\sup _{\tau \in \mathcal{T}^{P}} \mathbb{E}\left[\mathrm{e}^{-r \tau}\left(v_{G}\left(P_{\tau}^{p}\right)-I_{G}\right)\right]
$$

Note that, contrary to $v_{N}$, the payoff $v_{G}$ is non linear in the current output price. It is easy to check that the solution to this problem is to invest as soon as the output price 
$P$ exceeds the critical threshold:

$$
p_{G}= \begin{cases}\frac{\beta}{\beta-1} \frac{I_{G}(r-\mu)}{x_{G}} & \text { if } \frac{x_{N}}{x_{G}}-1<\frac{I_{N}}{I_{G}}, \\ \frac{\beta}{\beta-1} \frac{\left(I_{G}+I_{N}\right)(r-\mu)}{x_{N}} & \text { if } \frac{x_{N}}{x_{G}}-1 \geq \frac{I_{N}}{I_{G}} .\end{cases}
$$

In the first case, $p_{G}<p_{G N}$ and it is optimal to invest first in project $G$, and then to wait until $P$ exceeds $p_{G N}$ to invest in $N$. In that case, investment in $G$ is myopic in the sense that it occurs at the same time than if the option of ultimately switching to $N$ was not present, as in Leahy (1993). In the second case, $p_{G} \geq p_{G N}$ and the two investments are simultaneous.

We can use these computations to solve problem (1) in one special case.

Lemma 2.1 Suppose that it is the case that:

$$
\frac{I_{G}}{I_{N}} \geq \frac{x_{G}}{x_{N}} .
$$

Then the solution to (1) consists to invest in $N$ as soon as $P$ exceeds the threshold $p_{N}$, i.e., $V=V_{N}$.

Intuitively, what happens in that case is that the option of investing in $N$ is so attractive compared to that of investing in $G$ that the latter is never exercised: adding the option to invest in $G$ before investing in $N$ does not modify the investor's behavior. Economically, this corresponds to a situation in which there are increasing returns to scale in investment, with larger projects having larger average product, and we obtain the result of Dixit (1993) that it is then optimal to wait to invest in the larger project. To rule out this case, we shall henceforth assume that:

$$
\frac{I_{G}}{I_{N}}<\frac{x_{G}}{x_{N}} .
$$

Note that this implies that the optimal investment trigger $p_{G}$ in problem (3) lies below $p_{G N}$. Hence if the investor were constrained to invest in $G$ before than to invest in $N$, there will typically be a delay between the two investments. Furthermore, (4) also implies the tighter restriction that $p_{G}<p_{N}$.

It turns out that condition (4) is not sufficient to ensure that the problem is not degenerate, with the option of investing in $N$ always dominating that of investing in $G$. Indeed, it may be the case that at the optimal investment trigger $p_{G}$ for problem (3), the value of delaying investment until $p_{N}$ and invest in $N, V_{N}\left(p_{G}\right)$, exceeds that of investing in $G$ immediately, $v_{G}\left(p_{G}\right)-I_{G}$. The following lemma gives a necessary and sufficient condition under which this will be true. 
Lemma 2.2 Suppose that it is the case that:

$$
\left(\frac{x_{G}}{x_{N}}\right)^{\beta}\left(\frac{I_{G}}{I_{N}}\right)^{1-\beta}+\left(1-\frac{x_{G}}{x_{N}}\right)^{\beta} \leq 1 .
$$

Then the solution to (1) consists to invest in $N$ as soon as $P$ exceeds the threshold $p_{N}$, i.e., $V=V_{N}$.

Note that (4) is not inconsistent with the condition stated in the lemma. Hence, it is possible that (4) holds and still the option of investing in $N$ always dominates that of investing in $G$. To rule out this case, we shall henceforth assume that:

$$
\left(\frac{x_{G}}{x_{N}}\right)^{\beta}\left(\frac{I_{G}}{I_{N}}\right)^{1-\beta}+\left(1-\frac{x_{G}}{x_{N}}\right)^{\beta}>1 .
$$

Note that (5) implies (4) but not vice versa. Given (5), there will always be a region of the state space in which the option of investing in $G$ should be exercised before that of investing in $N$. It should be noted that (5) implies that $v_{G}\left(p_{G}\right)-I_{G}>v_{N}\left(p_{G}\right)-I_{N}$ and thus that $p_{G}<\tilde{p}$.

Note that if the option of investing in $N$ were not available once investment in $G$ is sunk, as in Dixit (1993), (5) would be modified to:

$$
\left(\frac{x_{G}}{x_{N}}\right)^{\beta}\left(\frac{I_{G}}{I_{N}}\right)^{1-\beta}>1 .
$$

In particular, if $\sigma$ goes to infinity and thus $\beta$ goes to 1 , this requirement would be violated as $x_{G}<x_{N}$. Thus, when the option to switch from $G$ to $N$ is not available, it is never optimal to invest in $G$ if the volatility $\sigma$ is high enough (see Dixit (1993)). When the option to switch from $G$ to $N$ becomes available as in our model, it is possible to find parameter values for $x_{G}, x_{N}, I_{G}$ and $I_{N}$ such that (5) will be satisfied even for very large values of $\sigma .^{2}$ Hence the option to switch from $G$ to $N$ qualitatively affects the impact of volatility on the optimal investment decision.

\section{The Main Result}

\subsection{Preliminaries}

First, we verify that the optimal stopping region for problem (1) is non-empty, and therefore that the problem has a solution. Then we derive a key property of the optimal stopping region.

\footnotetext{
${ }^{2}$ Note that if $\beta=1$, (5) becomes an equality. The derivative of the left-hand side of (5) with respect to $\beta$ at $\beta=1$ is $\left(\ln \left(x_{G} / x_{N}\right)-\ln \left(I_{G} / I_{N}\right)\right) x_{G} / x_{N}+\ln \left(1-x_{G} / x_{N}\right)\left(1-x_{G} / x_{N}\right)$ which is positive under (4) if $x_{G} / x_{N}$ is close enough to 1 , which implies the claim.
} 
Let us define the stopping region $\mathcal{S}=\{p \geq 0 \mid V(p)=v(p)\}$ for (1), as well as the stopping time $\tau_{S}=\inf \left\{t \geq 0 \mid P_{t}^{p} \in \mathcal{S}\right\}$. If $\mathcal{S}$ is non-empty, then $\tau_{S}$ is almost surely finite and $V(p)=\mathbb{E}\left[\mathrm{e}^{-r \tau_{S}} v\left(P_{\tau_{S}}^{p}\right)\right]$ so that $\tau_{S}$ is an optimal stopping time for $(1)$. We have the following result.

Proposition 3.1 The optimal stopping region $\mathcal{S}$ is non-empty.

The next proposition is key to our results.

Proposition 3.2 The indifference point $\tilde{p}$ does not belong to the optimal stopping region $\mathcal{S}$.

The proof of this result is technical, and relies on the Itô-Tanaka-Meyer formula (Karatzas and Shreve (1991, Theorem 3.7.1)). The intuition is that, because the payoff function $v=\max \left\{v_{G}-I_{G}, v_{N}-I_{N}\right\}$ is not differentiable at the indifference point $\tilde{p}$, with $v_{p}\left(\tilde{p}^{+}\right)>v_{p}\left(\tilde{p}^{-}\right)$, the investor is always better off delaying the investment when the current value of $P$ is $\tilde{p}$ rather than investing in either project. As shown below, this implies that whenever (5) hold, the optimal stopping region $\mathcal{S}$ is not connected, and the optimal investment strategy is not a trigger strategy.

This result does not depend on our assumption that the investor has the option to switch from $G$ to $N$. Indeed, a similar result holds if the project choice is irreversible, as in the model of Dixit (1993). ${ }^{3}$ This points out to the fact that the solution he providesinvest at $p_{k}$ in the project for which $x_{k}^{\beta} / I_{k}^{\beta-1}$ is the largest, and, for $p \geq p_{k}$, invest immediately in the project with the greatest net present value - is not correct, unless (6) is violated, in which case it is never optimal to invest in $G$. But as soon as investing in $G$ is sometimes optimal, the optimal investment strategy is not a trigger strategy, and there will be a region of values for the output price $P$ around the indifference point in which investment will be delayed.

\subsection{The Optimal Investment Region}

We are now ready to state and prove our main result.

Theorem 3.1 Suppose that (5) hold. Then:

(i) The optimal stopping region $\mathcal{S}$ for (1) is the union of two intervals $\left[p_{G}, p_{L}\right]$ and $\left[p_{R}, \infty\right)$, where $p_{L}<\tilde{p}<p_{R}$. If the current value of $P$ lies in $\left[p_{G}, p_{L}\right]$, it is optimal to invest in $G$, while if it lies in $\left[p_{R}, \infty\right)$, it is optimal to invest in $N$;

\footnotetext{
${ }^{3}$ In his model, the indifference point is given by $(r-\mu)\left(I_{N}-I_{G}\right) /\left(x_{N}-x_{G}\right)$, and the left and right derivatives of the payoff function at the indifference point are respectively given by $x_{G} /(r-\mu)$ and $x_{N} /(r-\mu)$. Again, this upward jump in the derivative of the payoff function implies that it is not optimal to invest around the indifference point.
} 
(ii) The value function $V$ is continuously differentiable on $\mathbb{R}_{++}$and satisfies the following variational inequalities:

$$
\begin{aligned}
\frac{1}{2} \sigma^{2} p^{2} V_{p p}+\mu p V_{p}-r V & \leq 0, \\
V & \geq v, \\
\left(\frac{1}{2} \sigma^{2} p^{2} V_{p p}+\mu p V_{p}-r V\right)(V-v) & =0 .
\end{aligned}
$$

In particular, $p_{L}$ and $p_{R}$ are characterized by the following value matching and smooth-pasting conditions:

$$
\begin{aligned}
V\left(p_{L}\right) & =v_{G}\left(p_{L}\right)-I_{G}, \\
V\left(p_{R}\right) & =v_{N}\left(p_{R}\right)-I_{N}, \\
V_{p}\left(p_{L}\right) & =v_{G p}\left(p_{L}\right), \\
V_{p}\left(p_{R}\right) & =v_{N p}\left(p_{R}\right) .
\end{aligned}
$$

The key insight of this proposition is that, for parameter values satisfying (5), the optimal investment strategy is not a trigger strategy, and the optimal investment region is dichotomous. It should be noted that Dixit's (1993) solution remains valid on the segment $\left[0, p_{G}\right]$ of the state space: it is optimal to wait until the output price exceeds $p_{G}$ to invest in $G$. However, our solution departs from his in that there is a range of prices $\left(p_{L}, p_{R}\right)$ around the indifference point in which it is optimal for the investor to wait in order to decide in which project to invest. Note that if project $N$ were not immediately available, it would be optimal to invest in $G$ in that region. Thus delay in the region $\left(p_{L}, p_{R}\right)$ reflects the added opportunity to invest in $N$. Moreover, it is not difficult to check that $p_{R}>p_{N}$, so that for values of $P$ in $\left(p_{N}, p_{R}\right)$, the investor chooses to delay investment although he would have invested immediately if only one project, $G$ or $N$, were available. This illustrates the interaction between the two investment options: the investor is willing to delay further investment in $N$ because he knows that he will have the option to invest in $G$ if the output price deteriorates too much. Note that this remains true even if, on the interval $[0, \tilde{p})$, the option of investing in $G$ uniformly dominates that of investing in $N$ when these two options are evaluated separately, i.e., $V_{N}<V_{G}$ on this price range.

The value function $V$ of the investor's problem coincides with $V_{G}$ on $\left[0, p_{L}\right]$, then takes off from $v$ on $\left(p_{L}, p_{R}\right)$ and touches down $v_{N}-I_{N}$ at the point $p_{R}$.

$$
\text { -Insert Figure } 1 \text { here- }
$$

On the interval $\left[0, p_{G}\right], V(p)$ is of the form $A p^{\beta}$, where:

$$
A=\frac{1}{p_{G}^{\beta}}\left(\frac{I_{G}}{\beta-1}+\left(\frac{p_{G}}{p_{G N}}\right)^{\beta} \frac{I_{N}}{\beta-1}\right) .
$$


Standard considerations imply that on the interval $\left[p_{L}, p_{R}\right], V(p)$ is of the form $B p^{\alpha}+$ $C p^{\beta}$, where $\alpha=1 / 2-\mu / \sigma^{2}-\sqrt{\left(1 / 2-\mu / \sigma^{2}\right)^{2}+2 r / \sigma^{2}}$ is the negative root of the quadratic equation $\xi(\xi-1) \sigma^{2} / 2+\mu \xi-r=0$. The coefficients $B$ and $C$, as well as the critical thresholds $p_{L}$ and $p_{R}$ can be found in principle by solving the value matching and smooth-pasting conditions (10)-(13), although no analytic solution is available.

\subsection{Discussion}

Examples of investment policies more complex than a simple trigger strategy abound in the literature. The model of entry and exit by Dixit (1989) generates a two-trigger strategy: the critical output price above which it is optimal to enter an industry is higher than the one below which it is optimal to exit from it. Similarly, Abel and Eberly (1996) show that the investment policy of a firm under costly reversibility is characterized by a range of inaction in which it is optimal neither to purchase nor to sell capital. However, a common feature of these two papers is that the lower boundary of the inaction region corresponds to a disinvestment decision while the upper boundary corresponds to an investment decision. By contrast, in our model both $p_{L}$ and $p_{R}$ correspond to an investment decision - albeit in different projects.

A striking feature of the optimal investment strategy is that it may be optimal to invest in $G$ after a drop in the output price $P$ if the current value of $P$ lies in $\left(p_{L}, p_{R}\right)$. Rational investment in a down market can also be triggered by other factors. Competition is one of them. Grenadier (1996) develops a model of strategic exercise of investment options in which competitors can simultaneously invest when the investment's value goes down in an attempt to avoid preemption. Décamps, Mariotti and Villeneuve (2001) show that a similar phenomenon can occur in a single decision maker context when the drift of the value process is not known to the investor ex ante. The decision to invest then depends on both the observed current value of the project and the beliefs about the unknown drift. This generates path dependency in the optimal investment strategy and it may be rational to invest after a drop in the current value of the investment project. As we have shown, this phenomenon can result from the interaction between several investment options.

Finally, although we have derived our results for the case of two alternative projects, it is conceptually straightforward to extend them to an arbitrary number of projects with sunk investment costs $I_{1}, \ldots, I_{K}$ and output flows $x_{1}, \ldots, x_{K}$ ranked in increasing order. The analogue of Proposition 3.2 is that it is never optimal to invest when the output price level corresponds to a point of non-differentiability of the upper envelope of the net present values of investing in each project. As soon as the largest scale investment option does not dominate all the others, the optimal investment strategy will be characterized by several disconnected inaction regions in which the investor waits in order to determine in which project to invest. 


\section{APPENDIX}

Proof of Lemma 2.1. It is clear from the expressions for $p_{N}$ and $p_{G}$ that $p_{N} \leq p_{G}$ whenever $I_{G} / I_{N} \geq$ $x_{G} / x_{N}$. Next, we check that, in that case, $p_{N}>\tilde{p}$. This amounts to verify that $v_{G}\left(p_{N}\right)-I_{G}<$ $v_{N}\left(p_{N}\right)-I_{N}$. Since $p_{G N}>p_{N}$, this is true whenever:

$$
\frac{x_{G}}{r-\mu} p_{N}+\left(\frac{p_{N}}{p_{G N}}\right)^{\beta} \frac{I_{N}}{\beta-1}-I_{G}<\frac{I_{N}}{\beta-1},
$$

or, equivalently:

$$
\frac{x_{G}}{x_{N}} \frac{\beta}{\beta-1}+\frac{1}{\beta-1}\left(1-\frac{x_{G}}{x_{N}}\right)^{\beta}-\frac{1}{\beta-1}<\frac{I_{G}}{I_{N}} .
$$

Whenever $I_{G} / I_{N} \geq x_{G} / x_{N}$, it is therefore sufficient to check that:

$$
\frac{x_{G}}{x_{N}} \frac{\beta}{\beta-1}+\frac{1}{\beta-1}\left(1-\frac{x_{G}}{x_{N}}\right)^{\beta}-\frac{1}{\beta-1}<\frac{x_{G}}{x_{N}}
$$

which is always true as $x_{G} / x_{N}<1$ and $\beta>1$. Hence $p_{G} \geq p_{N}>\tilde{p}$. Since $v_{G}-I_{G}<v_{N}-I_{N}$ on $(\tilde{p}, \infty)$, it is never optimal to stop and invest in $G$ whenever the current value of $P$ belongs to that region. Similarly, it is never optimal to stop and invest in $G$ whenever the current value of $P$ belongs to $[0, \tilde{p}]$, since this is dominated by investing in $G$ as soon as $P$ exceeds $p_{G}>\tilde{p}$. Hence, it is never optimal to invest in $G$, which implies the result.

Proof of Lemma 2.2. Given (4), the requirement that $V_{N}\left(p_{G}\right) \geq v_{G}\left(p_{G}\right)-I_{G}$ is equivalent to:

$$
\frac{I_{G}}{\beta-1}+\left(\frac{p_{G}}{p_{G N}}\right)^{\beta} \frac{I_{N}}{\beta-1} \leq\left(\frac{p_{G}}{p_{N}}\right)^{\beta} \frac{I_{N}}{\beta-1},
$$

or, equivalently:

$$
\left(\frac{x_{G}}{x_{N}}\right)^{\beta}\left(\frac{I_{G}}{I_{N}}\right)^{1-\beta} \leq 1-\left(1-\frac{x_{G}}{x_{N}}\right)^{\beta}
$$

which is the condition in the statement of the lemma. Two cases may occur. Suppose first that $p_{G}>\tilde{p}$. Then clearly it is never optimal to invest in $G$ whenever the current value of $P$ belongs to $\left(p_{G}, \infty\right)$ as the investor could secure a higher payoff by investing in $N$ in that region. Similarly, it is never optimal to stop and invest in $G$ whenever the current value of $P$ belongs to $\left[0, p_{G}\right]$, since this is dominated by investing in $N$ as soon as $P$ exceeds $p_{N}>p_{G}$. Hence the result. Suppose now that $p_{G} \leq \tilde{p}$. We first prove that $p_{N}>\tilde{p}$. Suppose the contrary. Then we have $v_{G}\left(p_{N}\right)-I_{G} \geq v_{N}\left(p_{N}\right)-I_{N}$. Since $p_{G N}>p_{N}$, this is true whenever:

$$
\frac{x_{G}}{r-\mu} p_{N}+\left(\frac{p_{N}}{p_{G N}}\right)^{\beta} \frac{I_{N}}{\beta-1}-I_{G} \geq \frac{I_{N}}{\beta-1},
$$

or, equivalently:

$$
1-\left(1-\frac{x_{G}}{x_{N}}\right)^{\beta} \leq \frac{x_{G}}{x_{N}} \beta-\frac{I_{G}}{I_{N}}(\beta-1)
$$


Putting together (14) and (15), we obtain that:

$$
\left(\frac{x_{G}}{x_{N}}\right)^{\beta}\left(\frac{I_{G}}{I_{N}}\right)^{1-\beta}+\frac{I_{G}}{I_{N}}(\beta-1)-\frac{x_{G}}{x_{N}} \beta \leq 0 .
$$

The left-hand side of (16) is decreasing in $I_{G} / I_{N}$ on $\left[0, x_{G} / x_{N}\right]$ and equals zero for $I_{G} / I_{N}=x_{G} / x_{N}$. Hence (16) is violated on the relevant range of parameters given by (4), and we get a contradiction. It follows that $p_{N}>\tilde{p}$, as claimed. Next, we show that $v_{G}-I_{G}<V_{N}$ on $\left(p_{G}, \tilde{p}\right)$. Since $p_{N}>\tilde{p}$, this is true whenever:

$$
\frac{x_{G}}{r-\mu} p+\left(\frac{p}{p_{G N}}\right)^{\beta} \frac{I_{N}}{\beta-1}-I_{G}-\left(\frac{p}{p_{N}}\right)^{\beta} \frac{I_{N}}{\beta-1}<0 .
$$

As a function of $p$, the left hand side has a slope given by:

$$
\frac{x_{G}}{r-\mu}+p^{\beta-1} I_{N} \frac{\beta}{\beta-1}\left(\frac{1}{p_{G N}^{\beta}}-\frac{1}{p_{N}^{\beta}}\right) .
$$

This is clearly decreasing in $p$ as $\beta>1$ and $p_{G N}>p_{N}$. Hence it is sufficient to show that this slope is non-positive at $p_{G}$. Simple computations using the values of $p_{G}, p_{N}$ and $p_{G N}$ show that this is equivalent to (14). To conclude, note that it is never optimal to invest in $G$ whenever the current value of $P$ belongs $\left(p_{G}, \tilde{p}\right)$ as waiting until $P$ exceeds $p_{N}$ secures a higher payoff.

Proof of Proposition 3.1. Since $r>\mu$, the process $\left\{\mathrm{e}^{-r t} P_{t}^{p} ; t \geq 0\right\}$ is a positive and continuous supermartingale. In particular, it has a last element, namely zero. Hence, by the Optional Sampling Theorem (Karatzas and Shreve (1991, Theorem 1.3.22)), $\mathbb{E}\left[\mathrm{e}^{-r \tau} P_{\tau}^{p}\right] \leq p$ for any stopping time $\tau \in \mathcal{T}^{P}$. Next, note that there exists positive constants $C_{1}$ and $C_{2}$ such that for each $p \geq 0, v(p) \leq C_{1} p+C_{2}$. We have therefore:

$$
\mathbb{E}\left[\mathrm{e}^{-r \tau} v\left(P_{\tau}^{p}\right)\right] \leq C_{1} \mathbb{E}\left[\mathrm{e}^{-r \tau} P_{\tau}^{p}\right]+C_{2} \leq C_{1} p+C_{2}
$$

for any stopping time $\tau \in \mathcal{T}^{P}$, and thus $V(p) \leq C_{1} p+C_{2}$. According to optimal stopping theory, the process $\left\{\mathrm{e}^{-r\left(t \wedge \tau_{S}\right)} V\left(P_{t \wedge \tau_{S}}^{p}\right) ; t \geq 0\right\}$ is a martingale (El Karoui (1981)). Now, suppose that $\mathcal{S}=\emptyset$. Then the process $\left\{\mathrm{e}^{-r t} V\left(P_{t}^{p}\right) ; t \geq 0\right\}$ is a martingale. Therefore, for any $t \geq 0$,

$$
V(p)=\mathbb{E}\left[\mathrm{e}^{-r t} V\left(P_{t}^{p}\right)\right] \leq C_{1} p \mathrm{e}^{-(r-\mu) t}+C_{2} \mathrm{e}^{-r t} .
$$

Since $t$ is arbitrary and $r>\mu$, it would follow that $V$ is identically equal to zero, which is a contradiction. Hence the result.

Proof of Proposition 3.2. Let us define the operator $\mathcal{A}$ by:

$$
\mathcal{A} g=\frac{1}{2} \sigma^{2} p^{2} g_{p p}+\mu p g_{p}-r g,
$$

and let $f=v_{N}-I_{N}-v_{G}+I_{G}$. By construction, $f$ is a difference of two convex functions which satisfies $f(\tilde{p})=0$ and $f_{p}(\tilde{p})>0$, and we have $v=v_{G}-I_{G}+\max \{f, 0\}$. The function $v_{G}-I_{G}$ is of class $\mathcal{C}^{2}$ on $\mathbb{R}_{++} \backslash\left\{p_{G N}\right\}$. Therefore, according to the generalized Itô's formula (Krylov $(1980, \S 2.10)$ ) 
and the Itô-Tanaka-Meyer formula (Karazas and Shreve (1991, Theorem 3.7.1)), we have, for every $t \geq 0$,

$$
\begin{aligned}
\mathbb{E}\left[\mathrm{e}^{-r t} v\left(P_{t}^{\tilde{p}}\right)\right]=v(\tilde{p}) & +\mathbb{E} \int_{0}^{t} \mathrm{e}^{-r s} \mathcal{A}\left(v_{G}-I_{G}\right)\left(P_{s}^{\tilde{p}}\right) \mathrm{d} s \\
& +\mathbb{E} \int_{0}^{t} \mathrm{e}^{-r s} \mathcal{A} f\left(P_{s}^{\tilde{p}}\right) \chi_{\left\{P_{s}^{\tilde{s}} \geq \tilde{p}\right\}} \mathrm{d} s+\frac{1}{2} f_{p}(\tilde{p}) \mathbb{E}\left[\mathrm{e}^{-r t} L_{t}^{\tilde{p}}\right]
\end{aligned}
$$

where $\left\{L_{t}^{\tilde{p}} ; t \geq 0\right\}$ is the local time for the continuous semimartingale $P$ at $\tilde{p}$. We treat each term on the right-hand side of this equation separately. For the first term, since $v_{G}-I_{G}$ is of class $\mathcal{C}^{2}$ on $\left(0, p_{G N}\right)$ there exists a positive constant $C_{1}$ such that $\left|\mathcal{A}\left(v_{G}-I_{G}\right)(p)\right| \leq C_{1}$ for $p \in\left(0, p_{G N}\right)$. For $p \in\left(p_{G N}, \infty\right), \mathcal{A}\left(v_{G}-I_{G}\right)(p)=r\left(I_{N}+I_{G}\right)-x_{N} p$. Hence, for every $t \geq 0$,

$$
\begin{aligned}
\left|\mathbb{E} \int_{0}^{t} \mathrm{e}^{-r s} \mathcal{A}\left(v_{G}-I_{G}\right)\left(P_{s}^{\tilde{p}}\right) \mathrm{d} s\right| & \leq \mathbb{E} \int_{0}^{t} \mathrm{e}^{-r s}\left|\mathcal{A}\left(v_{G}-I_{G}\right)\left(P_{s}^{\tilde{p}}\right)\right| \mathrm{d} s \\
& \leq \mathbb{E} \int_{0}^{t} \mathrm{e}^{-r s}\left(x_{N} P_{s}^{\tilde{p}}+r\left(I_{G}+I_{N}\right)+C_{1}\right) \mathrm{d} s \\
& =\frac{x_{N} \tilde{p}}{r-\mu}\left(1-\mathrm{e}^{-(r-\mu) t}\right)+\frac{r\left(I_{G}+I_{N}\right)+C_{1}}{r}\left(1-e^{-r t}\right) .
\end{aligned}
$$

Since $r>\mu$, it follows that:

$$
\mathbb{E} \int_{0}^{t} \mathrm{e}^{-r s} \mathcal{A}\left(v_{G}-I_{G}\right)\left(P_{s}^{\tilde{p}}\right) \mathrm{d} s=o(\sqrt{t}) .
$$

For the second term, note that there exists positive constants $C_{2}$ and $C_{3}$ such that $|\mathcal{A} f(p)| \leq C_{2} p+C_{3}$ for $p \geq \tilde{p}$. Hence, for every $t \geq 0$,

$$
\begin{aligned}
\left|\mathbb{E} \int_{0}^{t} \mathrm{e}^{-r s} \mathcal{A} f\left(P_{s}^{\tilde{p}}\right) \chi_{\left\{P_{s}^{\tilde{s}} \geq \tilde{p}\right\}} \mathrm{d} s\right| & \leq \mathbb{E} \int_{0}^{t} \mathrm{e}^{-r s}\left|\mathcal{A} f\left(P_{s}^{\tilde{p}}\right) \chi_{\left\{P_{s}^{\tilde{s}} \geq \tilde{p}\right\}}\right| \mathrm{d} s \\
& \leq \mathbb{E} \int_{0}^{t} \mathrm{e}^{-r s}\left(C_{2} P_{s}^{\tilde{p}}+C_{3}\right) \mathrm{d} s \\
& =\frac{C_{2} \tilde{p}}{r-\mu}\left(1-\mathrm{e}^{-(r-\mu) t}\right)+\frac{C_{3}}{r}\left(1-e^{-r t}\right) .
\end{aligned}
$$

Since $r>\mu$, it follows that:

$$
\mathbb{E} \int_{0}^{t} \mathrm{e}^{-r s} \mathcal{A} f\left(P_{s}^{\tilde{p}}\right) \chi_{\left\{P_{s}^{\tilde{p}} \geq \tilde{p}\right\}} \mathrm{d} s=o(\sqrt{t}) .
$$

For the third term, note that by the Itô-Tanaka-Meyer formula,

$$
\mathbb{E}\left[\mathrm{e}^{-r t}\left(P_{t}^{\tilde{p}}-\tilde{p}\right)^{+}\right]=\mu \mathbb{E} \int_{0}^{t} \mathrm{e}^{-r s} P_{s}^{\tilde{p}} \chi_{\left\{P_{s}^{\tilde{p}} \geq \tilde{p}\right\}} \mathrm{d} s-r \mathbb{E} \int_{0}^{t} \mathrm{e}^{-r s}\left(P_{s}^{\tilde{p}}-\tilde{p}\right)^{+} \mathrm{d} s+\frac{1}{2} \mathbb{E}\left[\mathrm{e}^{-r t} L_{t}^{\tilde{p}}\right] .
$$

The first two terms on the right-hand side of this equality can be shown to be on the order of $o(\sqrt{t})$ by the same reasoning as above. Hence:

$$
\frac{1}{2} \mathbb{E}\left[\mathrm{e}^{-r t} L_{t}^{\tilde{p}}\right]=\mathbb{E}\left[\mathrm{e}^{-r t}\left(P_{t}^{\tilde{p}}-\tilde{p}\right)^{+}\right]+o(\sqrt{t})
$$




$$
\begin{aligned}
& =\mathrm{e}^{-r t} \tilde{p}\left(\mathrm{e}^{\mu t} \mathbb{P}\left[W_{1} \leq \frac{\mu+\sigma^{2} / 2}{\sigma} \sqrt{t}\right]-\mathbb{P}\left[W_{1} \leq \frac{\mu-\sigma^{2} / 2}{\sigma} \sqrt{t}\right]\right)+o(\sqrt{t}) \\
& =\tilde{p} \sigma \sqrt{\frac{t}{2 \pi}}+o(\sqrt{t}),
\end{aligned}
$$

where the second and third equalities follow from a direct computation. We thus have:

$$
\mathbb{E}\left[\mathrm{e}^{-r t} v\left(P_{t}^{\tilde{p}}\right)\right]=v(\tilde{p})+\tilde{p} \sigma f_{p}(\tilde{p}) \sqrt{\frac{t}{2 \pi}}+o(\sqrt{t}) .
$$

Letting $t$ tend to zero and observing that $f_{p}(\tilde{p})>0$, we obtain that $\sup _{\tau \in \mathcal{T}^{P}} \mathbb{E}\left[\mathrm{e}^{-r \tau} v\left(P_{\tau}^{\tilde{p}}\right)\right]>v(\tilde{p})$. Hence $\tilde{p}$ does not belong to the optimal stopping region $\mathcal{S}$.

Proof of Theorem 3.1. Given (5), it follows that $\mathcal{S} \cap[0, \tilde{p}] \neq \emptyset$, otherwise one would have $V=V_{N}$, which is contradicted by the fact that $v_{G}\left(p_{G}\right)-I_{G}>V_{N}\left(p_{G}\right)$ under (5). Moreover, by Proposition 3.2 , inf $\mathcal{S}<\tilde{p}$. It is clear that for $p<p_{G}, V(p) \geq V_{G}(p)>v_{G}(p)-I_{G}$ so that $p_{G} \leq \inf \mathcal{S}$. We now prove the reverse inequality, establishing that $p_{G}=\inf \mathcal{S}$. We have:

$$
V\left(p_{G}\right)=\mathbb{E}\left[\mathrm{e}^{-r \tau_{S}}\left(v_{G}\left(P_{\tau_{S}}^{p_{G}}\right)-I_{G}\right)\right] \leq \sup _{\tau \in \mathcal{T}^{P}} \mathbb{E}\left[\mathrm{e}^{-r \tau}\left(v_{G}\left(P_{\tau}^{p_{G}}\right)-I_{G}\right)\right]=v_{G}\left(p_{G}\right)-I_{G},
$$

where the first equality follows from the fact that $\inf \mathcal{S}<\tilde{p}$, and the second from the definition of $p_{G}$. Since $V\left(p_{G}\right) \geq v_{G}\left(p_{G}\right)-I_{G}$, these quantities are in fact equal, and it follows that $p_{G} \geq \inf \mathcal{S}$, as claimed. A similar argument establishes that $\mathcal{S} \cap[0, \tilde{p}]$ is an interval $\left[p_{G}, p_{L}\right]$, and by Proposition 3.2 , $p_{L}<\tilde{p}$. We now prove that $\mathcal{S} \cap[\tilde{p}, \infty) \neq \emptyset$. If this was not the case, then $\mathcal{S}=\left[p_{G}, p_{L}\right]$, and for any $p>p_{L}$, we would have:

$$
V(p)=\mathbb{E}\left[\mathrm{e}^{-r \tau_{S}}\left(v_{G}\left(P_{\tau_{S}}^{p}\right)-I_{G}\right)\right]>v_{N}(p)-I_{N}
$$

or, equivalently:

$$
\left(\frac{p}{p_{L}}\right)^{\alpha}\left(\frac{x_{G}}{r-\mu} p_{L}+\left(\frac{p_{L}}{p_{G N}}\right)^{\beta} \frac{I_{N}}{\beta-1}-I_{G}\right)>\frac{x_{N}}{r-\mu} p-I_{N},
$$

where $\alpha=1 / 2-\mu / \sigma^{2}-\sqrt{\left(1 / 2-\mu / \sigma^{2}\right)^{2}+2 r / \sigma^{2}}<0$. For $p$ large enough, (17) is violated, and we get a contradiction. Let $p_{R}=\inf \mathcal{S} \cap[\tilde{p}, \infty)$. By Proposition 3.2, $p_{R}>\tilde{p}$. Moreover, it is immediate that $p_{R} \geq p_{N}$ otherwise waiting until $P$ exceeds $p_{N}$ to invest in $N$ would secure the investor a strictly greater payoff than investing in $N$ when the current value of $P$ is $p_{R}$. It then follows by an argument similar to the one used to prove that $p_{G}=\inf \mathcal{S}$ that $\mathcal{S} \cap[\tilde{p}, \infty)=\left[p_{R}, \infty\right)$. This concludes the proof of part (i).

To prove part (ii), note that the variational inequalities (10)-(13) are necessary and sufficient conditions to characterize the value function $V$ if we prove that $V$ is differentiable (Øksendal (2000, Theorem 10.4.1)). We need to do so only at the triggers $p_{G}, p_{L}$ and $p_{R}$. On $\left[0, p_{G}\right]$, we clearly have $V=V_{G}$, and the differentiability of $V$ at $p_{G}$ follows from a standard smooth-pasting argument for problem (3). We now prove that $V_{p}\left(p_{L}\right)=v_{G p}\left(p_{L}\right)$. Since $V \geq v_{G}-I_{G}$ and $V\left(p_{L}\right)=v_{G}\left(p_{L}\right)-I_{G}$, we have, for any $\varepsilon>0$,

$$
\frac{V\left(p_{L}+\varepsilon\right)-V\left(p_{L}\right)}{\varepsilon}=\frac{V\left(p_{L}+\varepsilon\right)-v_{G}\left(p_{L}\right)+I_{G}}{\varepsilon} \geq \frac{v_{G}\left(p_{L}+\varepsilon\right)-v_{G}\left(p_{L}\right)}{\varepsilon} .
$$


Following Karatzas and Shreve (1998, Lemma 7.8), let us introduce the stopping times $T_{\varepsilon}^{1}=\inf \{t \geq$ $\left.0 \mid\left(p_{L}+\varepsilon\right) H_{t} \leq p_{L}\right\}$ and $T_{\varepsilon}^{2}=\inf \left\{t \geq 0 \mid\left(p_{L}+\varepsilon\right) H_{t} \geq p_{R}\right\}$, where $H_{t}=\exp \left(\left(\mu-\sigma^{2} / 2\right) t+\sigma W_{t}\right)$ for each $t \geq 0$. The stopping time $T_{\varepsilon}=T_{\varepsilon}^{1} \wedge T_{\varepsilon}^{2}$ is optimal whenever the initial value of $P$ is $p_{L}+\varepsilon$. Therefore:

$$
V\left(p_{L}+\varepsilon\right)=\mathbb{E}\left[\mathrm{e}^{-r T_{\varepsilon}} v\left(\left(p_{L}+\varepsilon\right) H_{T_{\varepsilon}}\right)\right] \leq V\left(p_{L}\right)+\mathbb{E}\left[\mathrm{e}^{-r T_{\varepsilon}}\left(v\left(\left(p_{L}+\varepsilon\right) H_{T_{\varepsilon}}\right)-v\left(p_{L} H_{T_{\varepsilon}}\right)\right)\right],
$$

where the inequality follows from the fact that $T_{\epsilon}$ is not an optimal stopping time whenever the initial value of $P$ is $p_{L}$. The right-hand side of this inequality can be written as:

$V\left(p_{L}\right)+\left(v_{G}\left(p_{L}\right)-v_{G}\left(\frac{p_{L}^{2}}{p_{L}+\varepsilon}\right)\right) \mathbb{E}\left[\mathrm{e}^{-r T_{\varepsilon}} \chi_{\left\{T_{\varepsilon}=T_{\varepsilon}^{1}\right\}}\right]+\left(v_{N}\left(p_{R}\right)-v_{N}\left(\frac{p_{L} p_{R}}{p_{L}+\varepsilon}\right)\right) \mathbb{E}\left[\mathrm{e}^{-r T_{\varepsilon}} \chi_{\left\{T_{\varepsilon}=T_{\varepsilon}^{2}\right\}}\right]$.

Using standard results on the Laplace transform of the exit time of a Brownian motion on an interval (Karatzas and Shreve (1991, p. 100), together with the continuity of $v_{N}$, we obtain that:

$$
\left(v_{N}\left(p_{R}\right)-v_{N}\left(\frac{p_{L} p_{R}}{p_{L}+\varepsilon}\right)\right) \mathbb{E}\left[\mathrm{e}^{-r T_{\varepsilon}} \chi_{\left\{T_{\varepsilon}=T_{\varepsilon}^{2}\right\}}\right]=o(\varepsilon) .
$$

It follows that:

$$
\frac{V\left(p_{L}+\varepsilon\right)-V\left(p_{L}\right)}{\varepsilon} \leq \frac{v_{G}\left(p_{L}\right)-v_{G}\left(\frac{p_{L}^{2}}{p_{L}+\varepsilon}\right)}{\varepsilon}+o(1) .
$$

Using (18)-(19) and letting $\varepsilon$ go to zero, we obtain that $V_{p}\left(p_{L}^{+}\right)=v_{G p}\left(p_{L}^{+}\right)$, which implies the result. The proof of the differentiability of $V$ at $p_{R}$ is similar, and therefore omitted. 


\section{REFERENCES}

ABel, A.B., and J.C. EBerly (1996): "Optimal Investment with Costly Reversibility," Review of Economic Studies, 63, 581-593.

Arrow, K., and A.C. Fisher (1974): "Environmental Preservation, Uncertainty, and Irreversibility," Quarterly Journal of Economics, 88, 312-319.

Bar-Illan, A, and W.C. Strange (1998): "A Model of Sequential Investment," Journal of Economic Dynamics and Control, 22, 437-463.

DÉcamps, J.P., T. Mariotti, and S. Villeneuve (2001): "Investment Timing under Incomplete Information," Working Paper, Université de Toulouse.

Dixit, A.K. (1989): "Entry and Exit Decisions Under Uncertainty," Journal of Political Economy, 97, 620-638.

Dixit, A.K. (1993): "Choosing Among Alternative Discrete Investment Projects under Uncertainty," Economics Letters, 41, 265-268.

Dixit, A.K., and R.S. PINDYCK (1994): Investment under Uncertainty, Princeton, Princeton University Press.

El Karoui, N. (1981): "Les Aspects Probabilistes du Contrôle Stochastique," Ecole d'Eté de Probabilités de Saint-Flour IX, Lecture Notes in Mathematics 876, 74239, Berlin, Springer Verlag.

Farzin, Y.H., K.J.M. Huisman, and P.M. KorT (1998): "Optimal Timing of Technology Adoption," Journal of Economic Dynamics and Control, 22, 779-799.

Grenadier, S. (1996): "The Strategic Exercise of Options: Development Cascades and Overbuilding in Real Estates Markets," Journal of Finance, 51, 1653-1679.

Henry, C. (1974): "Investment Decisions Under Uncertainty: The Irreversibility Effect," American Economic Review, 64, 89-104.

KAndel, E., and N.D. PeArson (2002): "Option Value, Uncertainty, and the Investment Decision," Journal of Financial and Quantitative Analysis, 37, 341-374.

Karatzas, I., and S. Shreve (1991): Brownian Motion and Stochastic Calculus, Berlin, Springer-Verlag.

Karatzas, I., and S. Shreve (1998): Methods of Mathematical Finance, Berlin, Springer-Verlag.

Krylov, N.V. (1980): Controlled Diffusion Processes, Berlin, Springer-Verlag. 
LeAHy, J. (1993): "Investment in Competitive Equilibrium: The Optimality of Myopic Behavior," Quarterly Journal of Economics, 104, 1105-1133.

MAJD, S., and R.S. PindyCK (1987): "Time to Build, Option Value, and Investment Decisions," Journal of Financial Economics, 18, 7-27.

McDonald, R., and D. Siegel (1986): "The Value of Waiting to Invest," Quarterly Journal of Economics, 101, 707-727.

PindycK, R.S. (1988): "Irreversible Investment, Capacity Choice, and the Value of the Firm," American Economic Review, 78, 969-985. 


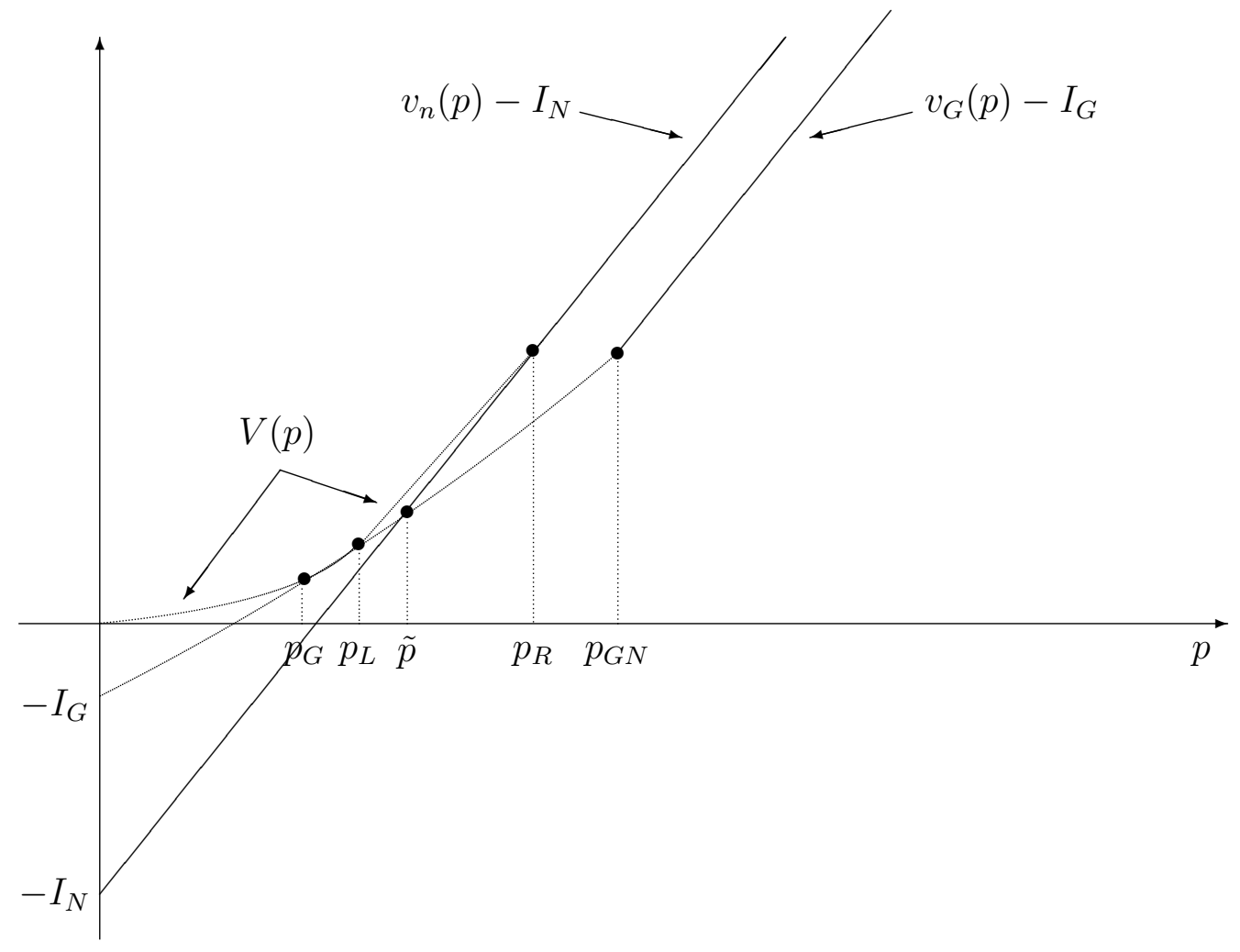

Figure 1: The Value Function $V$ 